\title{
A High Throughput Microplate Feeder Assay for Quantification of Consumption in Drosophila
}

\author{
Joshua D. Walters ${ }^{*}, 1$, Jeffrey S. Hatfield ${ }^{*}{ }^{1}$, Brandon B. Baker ${ }^{1}$, Trudy F. C. Mackay ${ }^{1}$, Robert R. H. Anholt ${ }^{1}$ \\ ${ }^{1}$ Department of Genetics and Biochemistry and Center for Human Genetics, Clemson University \\ *These authors contributed equally
}

\section{Corresponding Author}

Robert R. H. Anholt

ranholt@clemson.edu

\section{Citation}

Walters, J.D., Hatfield, J.S., Baker, B.B., Mackay, T.F.C., Anholt, R.R.H. A High

Throughput Microplate Feeder Assay for Quantification of Consumption in Drosophila. J. Vis. Exp. (172), e62771, doi:10.3791/62771 (2021).

\section{Date Published}

June 14, 2021

DOI

$10.3791 / 62771$

URL

jove.com/video/62771

\section{Abstract}

Quantifying food intake in Drosophila is used to study the genetic and physiological underpinnings of consumption-associated traits, their environmental factors, and the toxicological and pharmacological effects of numerous substances. Few methods currently implemented are amenable to high throughput measurement. The Microplate Feeder Assay (MFA) was developed for quantifying the consumption of liquid food for individual flies using absorbance. In this assay, flies consume liquid food medium from select wells of a 1536-well microplate. By incorporating a dilute tracer dye into the liquid food medium and loading a known volume into each well, absorbance measurements of the well acquired before and after consumption reflect the resulting change in volume (i.e., volume consumed). To enable high throughput analysis with this method, a 3D-printed coupler was designed that allows flies to be sorted individually into 96-well microplates. This device precisely orients 96- and 1536-well microplates to give each fly access to up to 4 wells for consumption, thus enabling food preference quantification in addition to regular consumption. Furthermore, the device has barrier strips that toggle between open and closed positions to allow for controlled containment and release of a column of samples at a time. This method enables high throughput measurements of consumption of aqueous solutions by many flies simultaneously. It also has the potential to be adapted to other insects and to screen consumption of nutrients, toxins, or pharmaceuticals.

\section{Introduction}

Drosophila melanogaster has seen wide use as a genetic model organism to study the biological underpinnings of food intake and traits associated with consumption ${ }^{1}$. It is estimated that $65 \%$ of human disease-causing genes have functional homologs in flies, with a significant proportion of those being expressed in functionally equivalent tissues between flies and humans ${ }^{2}$. Moreover, D. melanogaster's size, short intergenerational time, simple maintenance, and 
genetic tractability make it an attractive model for studies on the consumption of nutrients ${ }^{3,4}$ and toxicological and pharmacological effects of a variety of substances, including insecticides ${ }^{5}$, pollutants ${ }^{6}$, pharmaceuticals ${ }^{7}$, and drugs of abuse $^{8,9,10}$.

In many cases, the study of such traits requires precise quantification of consumption. Methods for quantifying consumption are diverse and include the CApillary FEeder (CAFE) assay ${ }^{11}$, the MAnual FEeding (MAFE) assay ${ }^{12}$,
Proboscis Extension Response (PER) assay ${ }^{13}$, tracer dye extraction $^{14,15}$, oligonucleotide tracer extraction ${ }^{16}$, and radio-isotope extraction ${ }^{5,17}$. Recent efforts have focused on enhancing the throughput of these assays, as in the Expresso assay $^{18}$ or the plate-based Whole Animal Feeding FLat (WAFFL) system ${ }^{19}$. Despite their utility, these assays can be complicated, costly, or labor-intensive, hindering their use in high throughput studies.

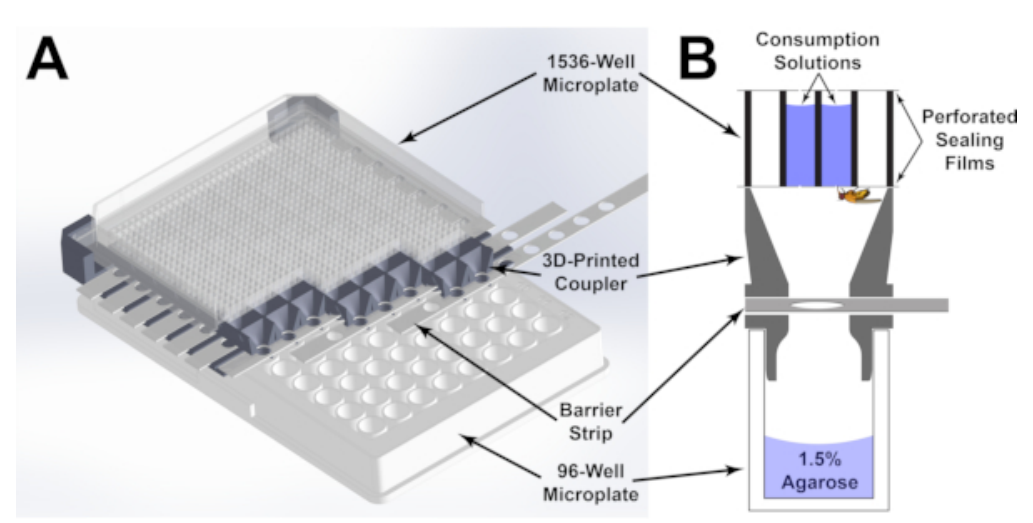

Figure 1: Components of the Microplate Feeder Assay. (A) 3D rendering of the assembled microplate feeder assay. The 1536-well microplate is oriented by the 3D-printed coupler such that each well of the lower 96-well microplate has access to four wells of the upper 1536-well microplate. Access to the wells can be controlled by adjusting the position of barrier strips slotted through the coupler. (B) A graphical representation of each well of the microplate feeder assay. Consumption solutions are retained in each well using a sealing film that has been perforated to allow access by the fly. Please click here to view a larger version of this figure. 


\section{Feeder Plate Preparation}

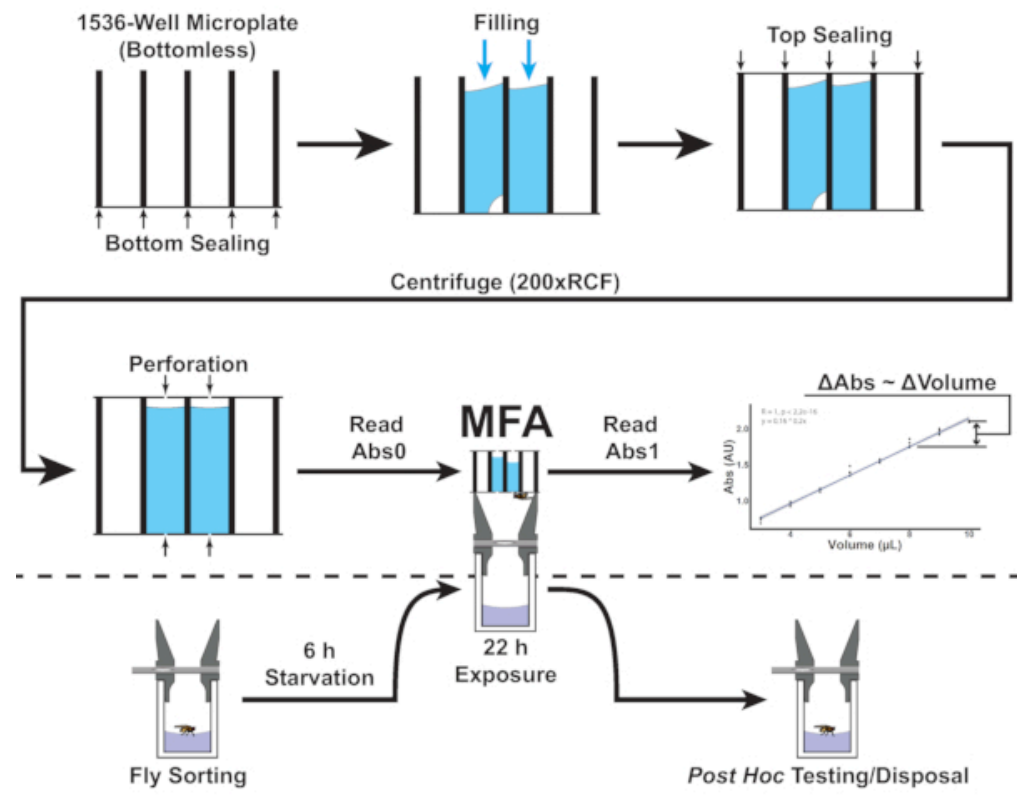

Starvation Plate Preparation

Figure 2: Overview of the procedures in the Microplate Feeder Assay. The figure shows a flow diagram that corresponds to steps 4.1-5.8 of the protocol. Please click here to view a larger version of this figure.

To overcome these hurdles, the Microplate Feeder Assay (MFA; Figure 1) was developed. In this assay, flies are housed individually in 96-well microplates. Each microplate is coupled to a 1536-well microplate using a custom, 3D-printed device. The device precisely orients the two plates such that each fly in its respective well of the 96-well plate has access to 4 wells of the 1536-well microplate. By using a bottomless 1536-well plate and sealing films, solutions are dispensed into select wells and perforated with precise $0.25 \mathrm{~mm}$ diameter needles to provide access to the flies. Critically, allowing consumption directly from a microplate allows for immediate absorbance-based measurements using a microplate reader. A dilute tracer dye is incorporated into the consumption medium, and the change in absorbance after exposure is used to determine the volume consumed (Figure 2 and
Figure 3). Since the liquid in each well approximates a column of fluid, volumetric differences will manifest as differences in the height of the column. (Figure 3A) According to the BeerLambert law ${ }^{20}$ :

$A=\varepsilon l c$

where $A$ is the absorbance, $\varepsilon$ is the molar absorption coefficient for the attenuating analyte, $I$ is the optical path length, and $c$ is the concentration of the attenuating analyte. Thus, with constant molar absorption coefficient and concentration, changes in absorbance are due solely to changes in the optical light path, i.e., the fluid level within a given well. By measuring absorbance before and after 
exposure, the proportional change in absorbance reflects the proportional change in volume (Figure 3B).
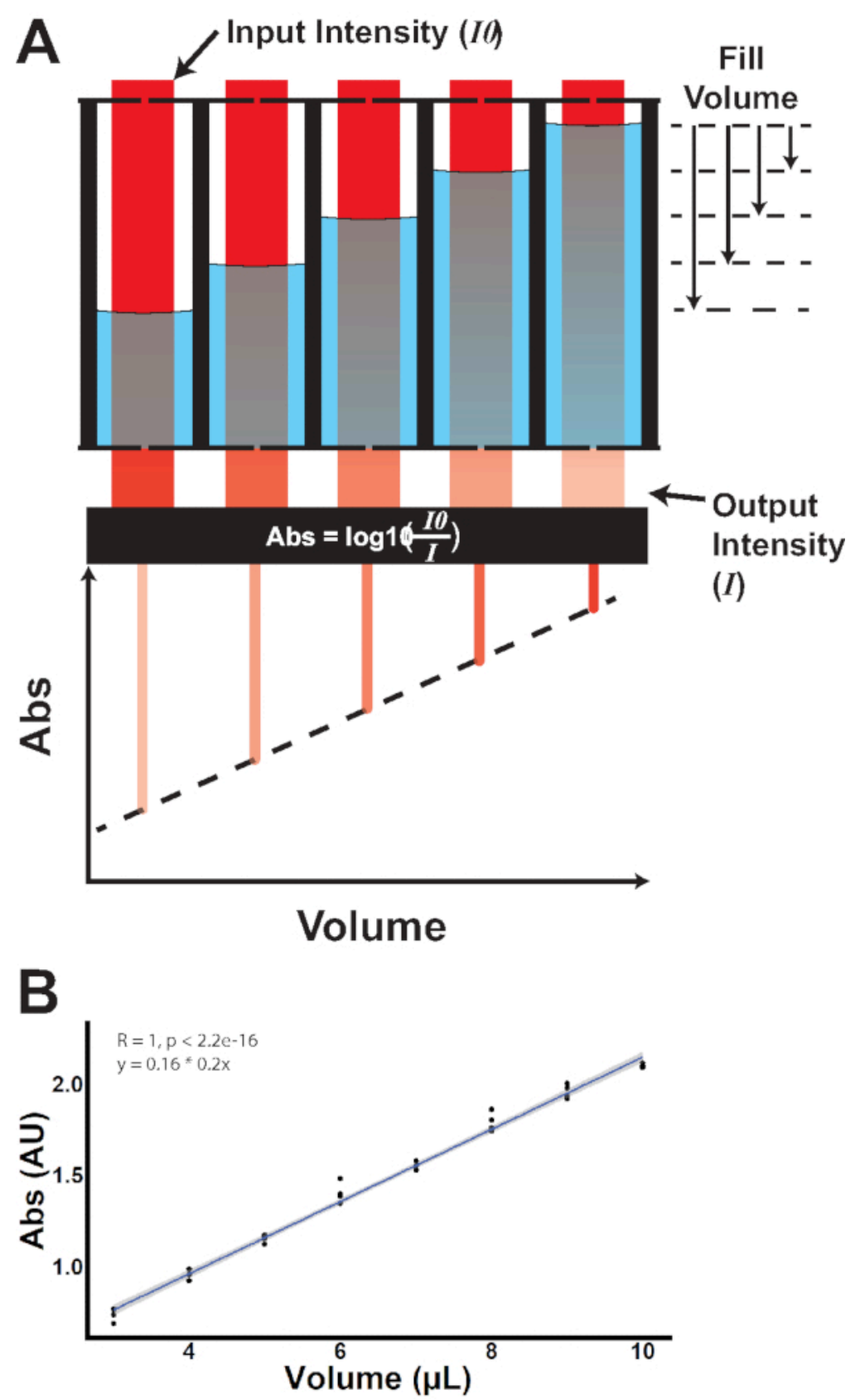

Figure 3: Absorbance-based quantification of well volume. (A) Incident light of known input intensity $\left(I_{0}\right)$ traverses each well. Attenuation of light at different fill volumes yields different output intensities $(I)$, exhibiting a linear relationship between volume and absorbance. (B) Empirical measurement of absorbance vs. volume. Please click here to view a larger version of this figure. 
Based on the change in volume, the amount of any ingested compound can be calculated from its known concentration in the feeding solution. The parts needed for the assay are low in cost and have a high degree of reusability, substantially reducing the recurring cost of the assay. Thus, this procedure offers an affordable, high throughput method of precisely quantifying consumption.

\section{Protocol}

\section{Starvation plate preparation}

1. Weigh out $1.5 \mathrm{~g}$ of agarose into a $250 \mathrm{~mL}$ glass beaker.

2. Add $100 \mathrm{~mL}$ of distilled $\mathrm{H}_{2} \mathrm{O}$ to the beaker.

3. Microwave intermittently until agarose is fully molten.

NOTE: Observe the beaker since the agarose is prone to boiling over.

4. Pour the molten agarose into a reagent trough and dispense $80 \mu \mathrm{L}$ of molten agarose into each well of a 96 well microplate using a multichannel pipette. Allow plates to cure while covered at room temperature. Refrigerate the leftover agarose for up to a week in a sealed bag and re-melt it for making additional plates.

\section{Fly sorting and starvation}

1. Prepare couplers by inserting barrier strips into the barrier strip channels. If barrier strips are too loose, coil them around the finger to give them curvature to hold them in the channels.

2. Affix the coupler to a starvation plate. Do not use the coupler to manipulate the plate since the coupler may slip off. Ensure that the couplers are correctly oriented (i.e., ensure the angled corner of the coupler matches the angled corner of the microplate).

3. Under $\mathrm{CO}_{2}$ anesthesia (Table of Materials), sort 3-5day old flies. Load individual flies by column into the starvation plate.

NOTE: Although flies are loaded by column, it is recommended to distribute groups of samples down rows of the plate rather than down columns (see Figure $\mathbf{4}$ for plate layout example).

4. Close each column as it fills by adjusting its barrier strip to the closed position. 


\begin{tabular}{|c|c|c|c|c|c|c|c|c|c|c|c|c|}
\hline \multicolumn{13}{|c|}{ Starvation Plate } \\
\hline 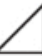 & 1 & 2 & 3 & 4 & 5 & 6 & 7 & 8 & 9 & 10 & 11 & 12 \\
\hline A & E & E & E & E & E & E & $E$ & $E$ & $E$ & $\mathbf{E}$ & $E$ & $E$ \\
\hline B & $E$ & E & E & E & E & E & $E$ & E & E & $\mathbf{E}$ & $E$ & E \\
\hline C & M & M & M & M & M & M & M & M & M & $M$ & $M$ & M \\
\hline D & M & M & M & M & M & M & M & M & M & M & M & M \\
\hline E & M & M & M & $\mathrm{M}$ & M & $M$ & M & M & M & M & M & M \\
\hline $\mathbf{F}$ & $\mathbf{F}$ & $\mathbf{F}$ & $\mathbf{F}$ & $\mathbf{F}$ & $\mathbf{F}$ & $\mathbf{F}$ & $\mathbf{F}$ & $\mathbf{F}$ & $\mathbf{F}$ & $\mathbf{F}$ & $\mathbf{F}$ & $F$ \\
\hline G & $F$ & $F$ & $\mathbf{F}$ & $\mathbf{F}$ & $\mathbf{F}$ & $\mathbf{F}$ & $\mathbf{F}$ & $\mathbf{F}$ & $F$ & $\mathbf{F}$ & $F$ & $F$ \\
\hline $\mathbf{H}$ & $F$ & $F$ & $F$ & $F$ & $F$ & $\mathbf{F}$ & $\mathbf{F}$ & $\mathbf{F}$ & $\mathbf{F}$ & $F$ & $F$ & $F$ \\
\hline
\end{tabular}

Figure 4: Representative Starvation Plate Layout. The diagram shows the organization of evaporation controls and male and female flies in a 96-well plate used in this study. Alternative configurations, including alternating rows of males and females with evaporation controls in rows $\mathrm{A}$ and $\mathrm{H}$, can also be used. Please click here to view a larger version of this figure.

5. Carefully record the sample layout within the microplate. Once the starvation plate is filled, allow the flies to recover spontaneously after removing the $\mathrm{CO}_{2}$ and starve them for $6 \mathrm{~h}$ starting from their initial anesthetization time.

\section{Liquid food preparation}

NOTE: Make liquid food fresh every day.

1. Prepare a $10 \mathrm{mg} / \mathrm{mL}$ of dye stock solution of FD\&C Blue $\# 1$ in distilled $\mathrm{H}_{2} \mathrm{O}$.

NOTE: This may be stored at room temperature for up to 6 months.

2. Prepare $10 \mathrm{~mL}$ of liquid food (4\% sucrose, $1 \%$ yeast extract, $40 \mu \mathrm{g} / \mathrm{mL}$ of FD\&C Blue \#1) in a $15 \mathrm{~mL}$ conical tube by dissolving $0.4 \mathrm{~g}$ of sucrose and $0.1 \mathrm{~g}$ of yeast extract in $10 \mathrm{~mL}$ of distilled $\mathrm{H}_{2} \mathrm{O}$. Vortex the tube until the solids fully dissolve. Add $40 \mu \mathrm{L}$ of dye stock solution and invert the tube repeatedly to homogenize the solution.

3. Transfer the liquid food into a $10 \mathrm{~mL}$ syringe tipped with a $0.45 \mu \mathrm{m}$ filter. Filter $\sim 1.5 \mathrm{~mL}$ of the solution at a time into a $1.7 \mathrm{~mL}$ microcentrifuge tube. Set aside the syringe containing the solution and filter the additional solution as needed during feeder plate preparation.

\section{Feeder plate preparation}

NOTE: Handle the feeder plates gently after filling to prevent the formation of bubbles or droplets in the well that could influence the absorbance readings. 
1. Prepare a feeder plate by sealing the bottom of a 1536well microplate with a sealing film. Use a sealing paddle to adhere to the film thoroughly. Trim excess film off the left and right edges with a razor blade.
2. Dispense $10 \mu \mathrm{L}$ of the filtered liquid food column-wise (see Figure 5 for illustration) into the appropriate wells of the 1536-well microplate. Dispense into the upper-lefthand well for each cluster of four wells (see Figure 5 for illustration).

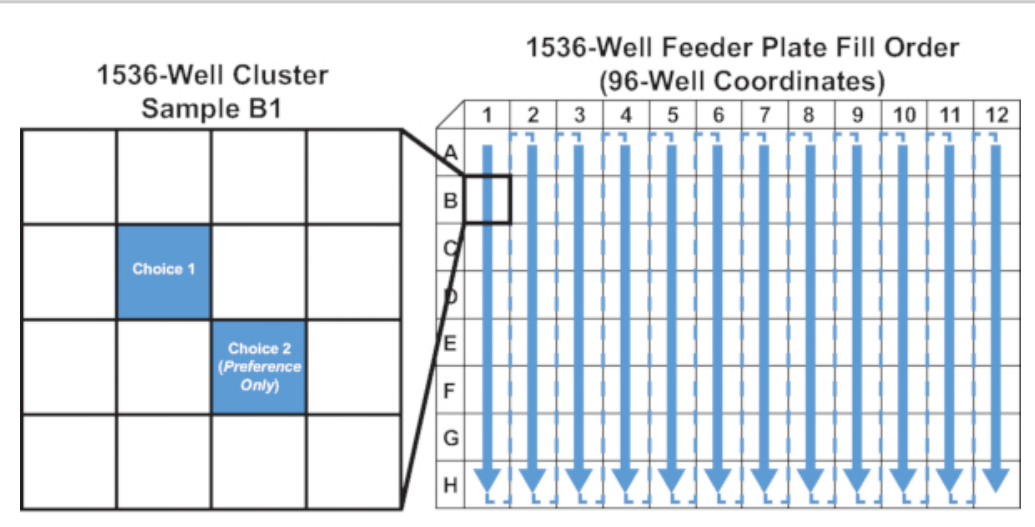

Figure 5: Fill order and well location for the 1536-well feeder plate. The diagram illustrates step 4.2 of the protocol. Arrows show the order in which the feeding solution is introduced in the feeder plate one column at a time from column 1 through 12. Sample B1 is magnified to show an example of the location of feeding solutions for 1-choice and 2-choice assays. Please click here to view a larger version of this figure.

3. Once all the wells are filled, apply a sealing film to the top of the plate. Use a sealing paddle to adhere to the film thoroughly. Trim excess film off the left and right edges with a razor blade. Repeat for the desired number of plates.

4. Centrifuge the plates at $200 \times g$ for $10 \mathrm{~s}$ to settle the fluid. Do not allow the plate to be chilled since this can cause condensation to build up in the wells, obscuring absorbance readings.

\section{Exposure}

1. Once the flies are ready for the consumption assay, perforate the wells on the top surface of the plate with the needle probe tool equipped with a $0.25 \mathrm{~mm}$ diameter needle. Use the same order to perforate as was used when dispensing the solutions. Flip the plate and perforate the wells on the bottom. Wipe off the needle between solutions to prevent cross-contamination. Be careful not to touch the perforations as this wicks the solution from the wells.

2. Read the plate's absorbance at $630 \mathrm{~nm}$ without a lid.

3. Place an internal lid on the top sealing film to ensure that the condensation rings encircle the perforated wells. Place the external lid on the plate.

4. Place the feeder plate face-up on the coupler such that the guides align the appropriate holes of the feeder plate and starvation plate. Ensure that the coupler and plates are correctly oriented (i.e., ensure the angled corner of 
the coupler and the plates match). Wrap elastic bands around the top and bottom plates to hold the coupler together. Check for alignment and gaps between the feeder plate and coupler.

5. Once all the feeder plates are loaded onto the couplers, open the wells for the plates by adjusting the barrier strips on the coupler. Place the coupler/plate assemblies in the secondary container. Each secondary container can accommodate up to six assemblies.

6. Place the lower half of a pipette box containing soaked paper towels into each secondary container to provide humidity. Close the lid of the secondary containers and transfer them to a controlled environment $\left(25^{\circ} \mathrm{C}\right.$, humidity controlled, $12 \mathrm{~h}$ light:dark cycles). Allow the flies to consume for $22 \mathrm{~h}$.

7. After the $22 \mathrm{~h}$ exposure, check each plate for dead flies and update the plate layout accordingly. After all the plates are checked, anesthetize the flies en masse by pumping $\mathrm{CO}_{2}$ inside the secondary container. After $\sim 60$ $\mathrm{s}$, ensure that all the flies are immobilized. Gently tamp the flies into the starvation plate and replace the plastic barrier strips. Remove the feeder plates for reading.

8. Re-read the plate's absorbance at $630 \mathrm{~nm}$. Repeat until all plates have been read.

\section{Data analysis}

NOTE: Analysis can be performed with the investigator's preferred software package.

1. Omit any flies that died during the $22 \mathrm{~h}$ exposure.

2. For each well, calculate the volume consumed as:

$$
C=V *\left(\frac{A B S_{0}-A B S_{1}}{A B S_{0}}\right)
$$

C - Volume Consumed $(\mu \mathrm{L})$

$V$ - Initial Well Volume (i.e., $10 \mu \mathrm{L}$ )

$A B S_{O}$ - Pre-Exposure Absorbance

$A B S_{1}$ - Post-Exposure Absorbance

NOTE: Consumption is denoted as a positive volume in the calculation.

3. To account for evaporation, subtract the mean evaporation volume from fly consumption values within respective plates. For 2-choice/preference testing, adjust every well by its respective solution, e.g., adjust "choice 1 " wells by "choice 1" in the evaporation controls.

4. After adjusting for evaporation, drop any samples with a consumption value less than zero.

5. For 2-choice testing, calculate the preference for each well as:

$P=\frac{F_{A}-F_{N}}{F_{A}+F_{N}}$

$P$ - Preference Index (positive direction indicates preference)

$F_{A}$ - Volume of Liquid Food Consumed Containing Additive (Choice 2)

$F_{N}$ - Volume of Normal Liquid Food Consumed (Choice 1)

\section{Microplate and coupler washing protocol.}

NOTE: Take care to prevent damage to the bottoms of the microplates, as damage can affect sealing.

1. Remove the films and labels from the 1536-well microplates. Separate the couplers and barrier strips. Place the barrier strips in a sealable container, such as a bottle. Wash the barrier strips by vigorously shaking in a 
series of warm tap water, mild detergent solution, warm tap water, and then distilled $\mathrm{H}_{2} \mathrm{O}$.

2. Rinse 1536-well microplates and couplers under warm tap water. For microplates, run tap water through each microplate's wells to clear as much solution and debris as possible. If needed, use a pipette tip to dislodge debris; do not use metal or glass utensils on the plates.

3. Cover each plate and coupler with a mild detergent solution (e.g., 1\% v/v Aquet). For the plates, scrub the surfaces with a gloved hand. For the couplers, use a brush.

4. Rinse each plate thoroughly with tap water, and then with distilled $\mathrm{H}_{2} \mathrm{O}$. Ensure that the wells are specifically rinsed out under the water flow.

5. Allow the plates and couplers to air dry covered at room temperature. Store in a clean storage bin until use.
NOTE: Never handle the 1536-well microplates without gloves. Residual oils from the skin can hinder sealing, leading to well leakage and evaporation.

\section{Representative Results}

To determine whether any correlations exist among the wells of individual plates, the evaporation was quantified for every well ( $n=96$ wells/plate for three plates). Evaporation was found to be $-0.036 \mu \mathrm{L} \pm 0.003 \mu \mathrm{L}$ (mean \pm SEM throughout). (Figure 6A) Pearson correlations were calculated to evaluate trends between evaporation and well locations. The correlation coefficient (Figure 6B,C) for evaporation versus rows was $-0.04(p=0.4949)$ and for evaporation vs columns was $-0.23(p=0.0001)$. Groups were subsequently distributed among columns to mitigate the mild but statistically significant correlations across columns. 

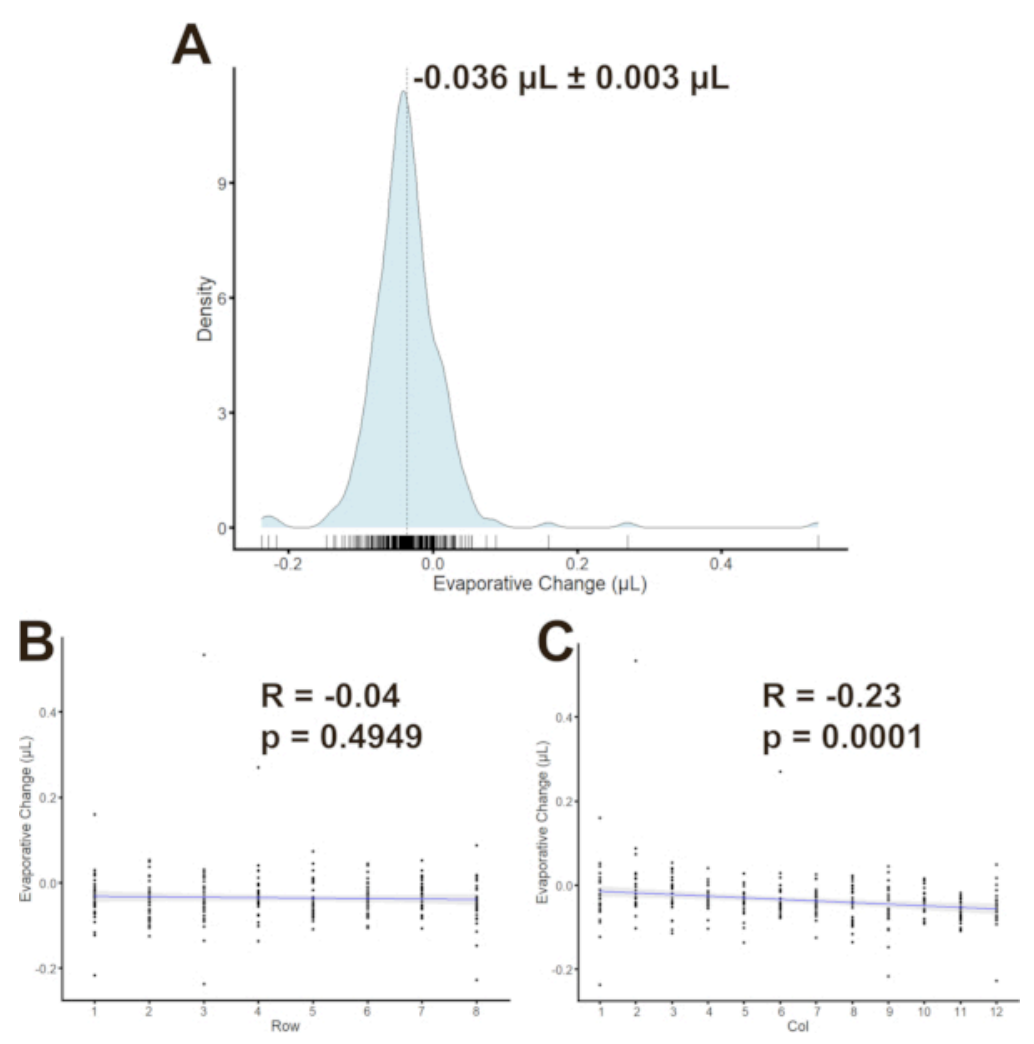

Figure 6: Evaporation in the MFA. (A) Density distribution of evaporation changes with mean \pm SD indicated by the dashed line. Correlations between evaporation and rows $(\mathbf{B})$ or columns $(\mathbf{C})$ with the Pearson correlation coefficient and p-value as indicated. Please click here to view a larger version of this figure.

To establish the validity of the protocol, consumption was quantified for 3-5-day old Canton-S B flies ( $n=36 /$ sex/plate and $n=24$ evaporation controls/plate for three plates) (Figure 7). Evaporation among the control wells was significantly different from zero $\left(-0.030 \mu \mathrm{L} \pm 0.006 \mu \mathrm{L}, \mathrm{p}=4.81 \times 10^{-6}\right.$; one sample $t$-test vs zero). Two samples were omitted (both male) from the dataset, one due to death during the overnight exposure and the other due to negative consumption value following adjustment for evaporation. This yielded a $>99 \%$ sample retention rate. 

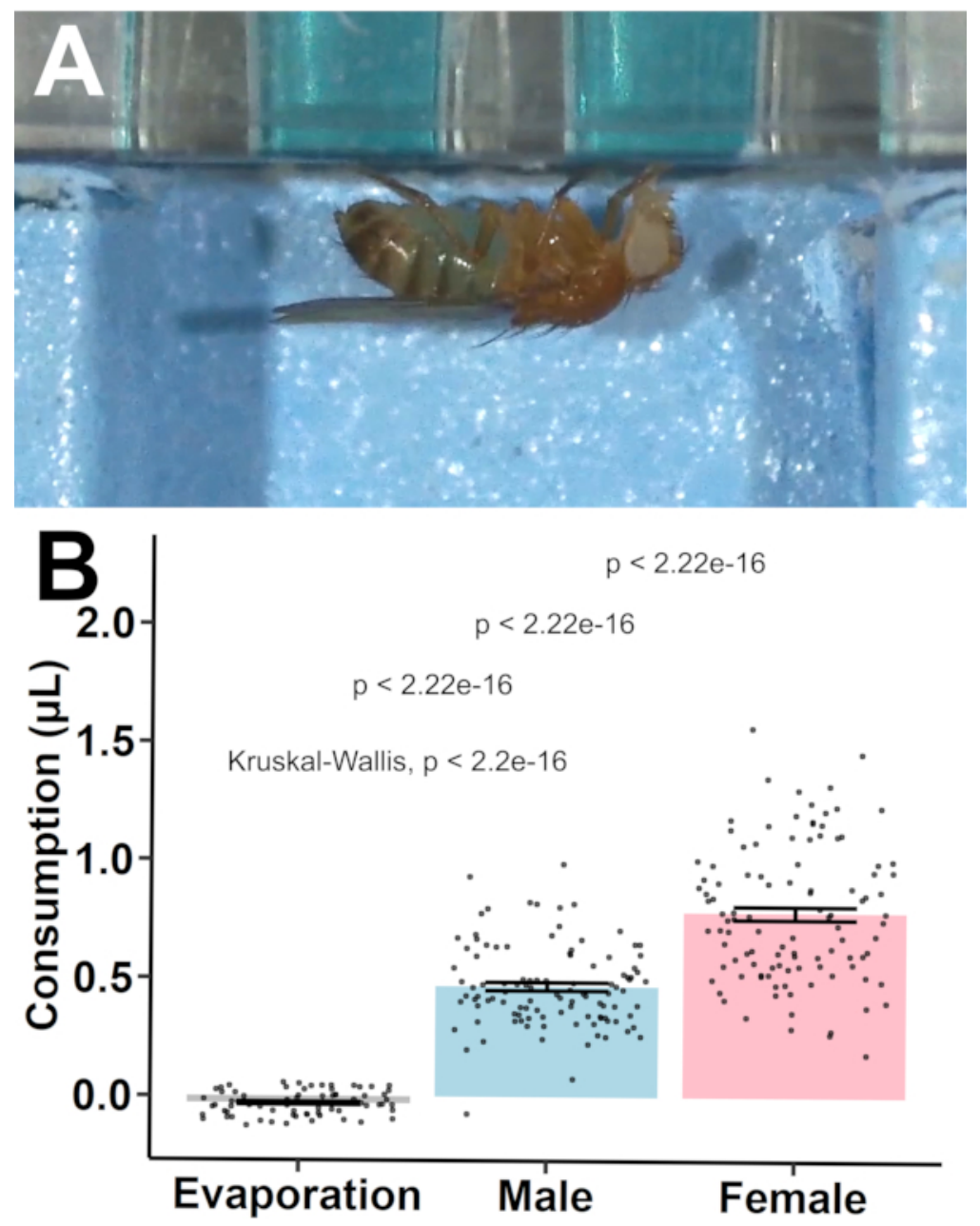

Figure 7: Consumption quantification using the MFA. (A) Consumption was visualized using a custom fabricated glass chamber. Flies were observed drinking from perforated wells and exhibited blue abdominal staining following ingestion of the dyed solution. See also Supplementary Video S.1. (B) Consumption values (mean \pm SEM) among evaporation controls, male flies, and female flies. Pairwise post hoc $t$-test with unequal variance was performed for statistical comparisons, with significance indicated by bars. Please click here to view a larger version of this figure.

Subsequently, an Analysis of Variance (ANOVA) model was constructed as described by $\mathrm{Y}=\mu+\mathrm{S}+\mathrm{P}+\mathrm{SxP}+\mathrm{e}$, with $\mathrm{Y}$ as the group mean, $\mu$ as the overall mean, $S$ as the fixed effect of sex, $\mathrm{P}$ as the fixed effect of plate, SxP as the interaction between Sex and Plate, and e as the residual variability. ANOVA showed no significant plate-to-plate variability $(p=$ $0.671)$ or sex-specific interactions with plates $(p=0.104)$ for consumption, while sex alone significantly contributed to the observed variation in consumption $\left(p=4.17 \times 10^{-18}\right)$. A post hoc $t$-test showed that males consumed significantly less than females $(0.500 \mu \mathrm{L} \pm 0.017 \mu \mathrm{L}$ vs $0.811 \mu \mathrm{L} \pm 0.028 \mu \mathrm{L}, \mathrm{p}=$ $1.13 \times 10^{-17}$, two sample $t$-test with unequal variance).

To demonstrate that the assay can be used for two-choice preference quantification, flies were given a choice between 
a $4 \%$ sucrose solution with $1 \%$ yeast extract and a $4 \%$ sucrose solution supplemented with $15 \%$ ethanol and $1 \%$ yeast extract. Both males and females showed overwhelming preference for the solution with ethanol and yeast extract with preference indices of $0.974 \pm 0.026$ for males and $0.876 \pm$ 0.06 for females (average \pm SEM) (Figure 8).
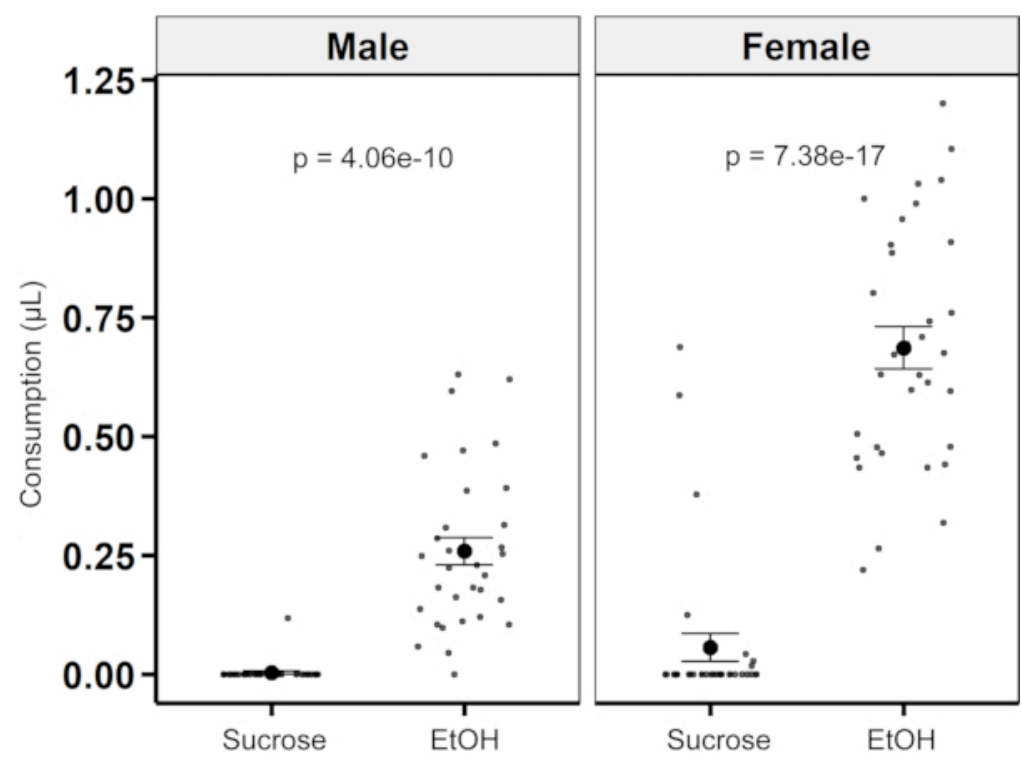

Figure 8: Preference quantification using the MFA. Consumption of $4 \%$ sucrose versus $4 \%$ sucrose supplemented with $15 \%$ ethanol and yeast extract for male and female flies ( $n=33$ for each sex). Male flies consumed more of the ethanol solution than the control sucrose solution $\left(0.511 \mu \mathrm{L} \pm 0.029 \mu \mathrm{L}\right.$ versus $0.00 \mu \mathrm{L} \pm 0.017 \mu \mathrm{L} ; \mathrm{p}=4.06 \mathrm{e}^{-10}$; two-sample $t$-test). Female flies also consumed more ethanol solution than the control sucrose solution $(0.939 \mu \mathrm{L} \pm 0.044 \mu \mathrm{L}$ versus $0.132 \mu \mathrm{L} \pm$ $0.044 \mu \mathrm{L} ; \mathrm{p}=7.38 \mathrm{e}^{-17}$; two-sample $t$-test). Please click here to view a larger version of this figure.

\section{Supplementary Video S.1: The video shows a fly} feeding from the perforated well and accumulating blue abdominal staining while ingesting the dyed solution. A still image is shown in Figure 7A. Please click here to download this video.

\section{Supplementary File S.2: Microplate Feeder Assay} Coupler. This is a 3D-printable construct of the coupler used in the MFA. Printing material Nylon PA12 was used for the MFA. Please click here to download this file.
Supplementary File S.3: Microplate Feeder Assay Barrier Strip. This contains the design of the plastic barrier strips used to toggle exposure of flies to the feeder plate. A single coupler can utilize up to twelve barrier strips. Please click here to download this file.

\section{Supplementary File S.4: Unpacking and fabrication} instructions for the Microplate Feeder Assay. Instructions are included for unpacking the coupler and the barrier strips. Fabrication instructions are included for the inner lid, outer 
lid, and secondary container used to limit evaporation during exposure. Please click here to download this file.

\section{Supplementary File S.5:Cost comparison of the} Microplate Feeder Assay (MFA) and a 1-choice single fly CApillary FEeder (CAFE) assay. Testing 72 flies/sex for a single line would require two sets of MFA equipment (couplers + plates + barrier strips), while the CAFE would only need 1 capillary for each culture vial. Despite the large difference in initial investment for the MFA, the large difference in recurring costs ( $\$ 14.80$ vs $\$ 46.08$, respectively) would allow for the upfront costs to be recovered after testing only 4 lines (breakeven point). Please click here to download this file.

\section{Discussion}

The study describes a novel protocol for quantifying consumption in Drosophila: the Microplate Feeder Assay (MFA). In this assay, flies consume from sealed wells of a 1536-well microplate through controlled-size perforations

(Figure 1, Figure 2; Supplementary Video S.1). Since liquid food is dyed and provided via microplate, measurements of the optical absorbance of the food can be obtained using a microplate spectrophotometer (Figure 3). In this manner, consumption is determined by comparing the absorbance before and after consumption, and then applying this proportion to the known volume dispensed prior to consumption. This was verified empirically by measuring the absorbance of different volumes of the dyed medium (Figure 3B).

To develop this assay, a device was needed that could leverage the absorbance-based quantification of consumption. Testing flies in a microplate format is appealing because it complements the microplate used to dispense food and allows for flexibility in selecting from multiple plate formats (e.g., 6-, 12-, 48-, or 96-well formats) by adjusting the coupler geometry. A 96-well microplate format was chosen to allow for individual fly culture.

The 3D printed device (Figure 1) precisely orients the 1536-well feeder plate with the 96-well culture plate, giving each fly access to up to 4 wells of the feeder plate for consumption. Furthermore, to provide adequate time for distributing flies into the housing plate and to control assay initiation, the device includes toggling barrier strips containing the flies in their respective wells and preventing breaches. The files needed to procure or modify these parts are provided (Supplementary Files S.2-S.3), as well as the necessary fabrication instructions for the relevant pieces

\section{(Supplementary File S.4).}

The MFA provides a simple high throughput method that complements more elaborate methods to monitor Drosophila feeding behavior ${ }^{18,21,22}$. The MFA offers multiple advantages over other methods used to quantify food intake. The throughput is increased by quantifying consumption using a plate reader. This eliminates manual measurements and obviates manual data entry. Data are also amenable to programmatic extraction and processing. In addition, the higher throughput increases the feasible number of biological replicates, particularly compared to communal feeder designs, which substantially increases the power to detect small differences in consumption. Using the MFA, a single experimenter can quantify the consumption or preference of over 500 flies per overnight run of the assay. By overlapping runs of the assay, over 2,000 flies can be tested in a 5-day period. Lastly, there are long-term cost savings due to the reusability of microplates and couplers (Supplementary File S.5). Using the MFA, the estimated cost per assay can be as low as $\$ 14.80$, with a $\$ 127.60$ up-front cost for the equipment. Using the classic CApillary FEeder (CAFE) assay, 
which requires costly precision microcapillaries, the estimated cost per assay for a comparable number of replicates is $\$ 46.08$. Thus, while there is an upfront investment in acquiring the necessary equipment, the reduction in recurring costs can lead to substantial savings, particularly in instances where repeated testing is performed.

As with all assays, the MFA has certain limitations. Chiefly, it requires access to a microplate spectrophotometer capable of reading 1536-well microplates. Additionally, the reliance on absorbance measurements for quantification makes the method susceptible to optical interference. This manifests as negative consumption values for a small subset of samples tested. Nutrients, drugs, pharmaceuticals, or toxins of interest must also be water-soluble to be compatible with the assay.

Despite its limitations, this method offers a high throughput method of quantifying consumption behaviors in Drosophila. Furthermore, the coupling device could be easily modified to accept many plate formats, allowing it to accommodate a variety of insect species.

\section{Disclosures}

The authors have no conflicts of interest to disclose.

\section{Acknowledgments}

This work was supported by a grant from the National Institute on Drug Abuse (U01 DA041613) to TFCM and RRHA.

\section{References}

1. Wong, R., Piper, M. D. W., Wertheim, B., Partridge, L. Quantification of food intake in Drosophila. PLOS ONE. 4 (6) (2009).
2. Ugur, B., Chen, K., Bellen, H. J. Drosophila tools and assays for the study of human diseases. Disease Models \& Mechanisms. 9 (3), 235-244 (2016).

3. Spitaler, U. et al. Yeast species affects feeding and fitness of Drosophila suzukii adults. Journal of Pest Science. 93 (4), 1295-1309 (2020).

4. Wang, Q. P. et al. PGC1a controls sucrose taste sensitization in Drosophila. Cell Reports. 31 (1), 107480 (2020).

5. Valtierra-de-Luis, D. et al. Quantification of dosemortality responses in adult Diptera: Validation using Ceratitis capitata and Drosophila suzukii responses to spinosad. PLoS ONE. 14 (2), 1-11 (2019).

6. Williams, M. J. et al. Exposure to bisphenol A affects lipid metabolism in Drosophila melanogaster. Basic \& Clinical Pharmacology \& Toxicology. 114 (5), 414-420 (2014).

7. Jajoo, A., Donlon, C., Shnayder, S., Levin, M., McVey, M. Sertraline induces DNA damage and cellular toxicity in Drosophila that can be ameliorated by antioxidants. Scientific Reports. 10 (1), 1-12 (2020).

8. Fochler, S. et al. Genetics of alcohol consumption in Drosophila melanogaster. Genes, Brain and Behavior. 16 (7), 675-685 (2017).

9. Highfill, C. A., Baker, B. M., Stevens, S. D., Anholt, R. R. H., Mackay, T. F. C. Genetics of cocaine and methamphetamine consumption and preference in Drosophila melanogaster. PLOS Genetics. 15 (5), e1007834 (2019).

10. Keebaugh, E. S., Park, J. H., Su, C., Yamada, R., Ja, W. W. Nutrition Influences caffeine-mediated sleep loss in Drosophila. Sleep. 40 (11) (2017). 
11. Ja, W. W. et al. Prandiology of Drosophila and the CAFE assay. Proceedings of the National Academy of Sciences. 104 (20), 8253-8256 (2007).

12. Qi, W. et al. A quantitative feeding assay in adult Drosophila reveals rapid modulation of food ingestion by its nutritional value. Molecular Brain. 8 (1), 87 (2015).

13. Shiraiwa, T., Carlson, J. R. Proboscis extension response (PER) assay in Drosophila. Journal of Visualized Experiments: JoVE. 3, 193 (2007).

14. Shell, B. C. et al. Measurement of solid food intake in Drosophila via consumption-excretion of a dye tracer. Scientific Reports. 8 (1), 1-13 (2018).

15. $\mathrm{Wu}, \mathrm{Q}$. et al. Excreta quantification (EX-Q) for longitudinal measurements of food intake in Drosophila. iScience. 23 (1), 100776 (2020).

16. Park, A., Tran, T., Atkinson, N. S. Monitoring food preference in Drosophila by oligonucleotide tagging. Proceedings of the National Academy of Sciences of the United States of America. 115 (36), 9020-9025 (2018).

17. Deshpande, S. A. et al. Quantifying Drosophila food intake: Comparative analysis of current methodology. Nature Methods. 11 (5), 535-540 (2014).

18. Yapici, N., Cohn, R., Schusterreiter, C., Ruta, V., Vosshall, L. B. A Taste circuit that regulates ingestion by integrating food and hunger signals. Cell. 165 (3), 715-729 (2016).

19. Jaime, M. D. L. A. et al. The high-throughput WAFFL system for treating and monitoring individual Drosophila melanogaster adults. bioRxiv. (2018).

20. IUPAC Compendium of Chemical Terminology (The "Gold Book"). Blackwell Scientific Publications. Research Triangle Park, NC (1997).
21. Itskov, P. M. et al. Automated monitoring and quantitative analysis of feeding behaviour in Drosophila. Nature Communications. 5, 4560 (2014).

22. Ro, J., Harvanek, Z. M., Pletcher, S. D. FLIC: highthroughput, continuous analysis of feeding behaviors in Drosophila. PLoS One. 9 (6), e101107 (2014). 